\title{
Percutaneous Endovascular Repair and Savage of Vascular Stream After Spontaneous and/or Iatrogenic Complications of Kidney Transplantation
}

\author{
Emanuela de Cillis ${ }^{1}$, Alessandro S. Bortone ${ }^{1, *}$, Pasquale Ditonno ${ }^{2}$, Lucio Garofalo ${ }^{2}$, Cinzia Cicala $^{1}$ \\ Michele Battaglia $^{2}$ and Luigi de Luca Tupputi Schinosa ${ }^{1}$ \\ ${ }^{1}$ Institute of Cardiac Surgery, ${ }^{2}$ Institute of Urology, University of Bari, Bari, Italy
}

\begin{abstract}
Background: Vascular complications are uncommon (1-3\%) in kidney transplantation. Recipient morbidities may result in loss of the allograft. Early and middle-term vascular complications include external iliac artery iatrogenic or spontaneous lesions and drainage vein thrombosis. Percutaneous transluminal angioplasty (PTA) is proposed as a valid, less invasive and alternative treatment to surgical repair.

Material and Methods: Three patients, in the last two years, referred to our Institute: one affected by dissection secondary to the site clamp and suture stenosis of the external iliac artery, one spontaneous pseudoaneurysm with uncontained rupture of the same artery and another with pseudoaneurysm at the site of previous transplantation. All patients were treated by endovascular PTA approach. Patients with pseudo-aneurysm were treated by self-expandable stent-graft PTFE endoprostheses (Hemobahn-Gore) release whereas in the other a balloon-expandable covered stent (Advanta V12 Boston Scientific) was used.

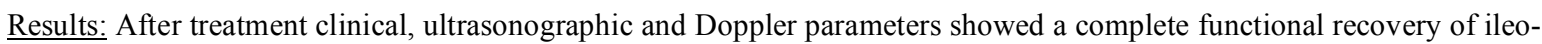
femoral axis as well renal function. All patients were discharged 5 days after the procedure. At an average follow-up of 12 months, by angio-CT scan, no recurrence of stenoses or aneurysms were found.

Conclusion: PTA followed by stent placement is safe, effective and less invasive than surgical repair. Short and middleterm follow-up showed the benefit of the procedure, moreover the hospital stay is dramatically reduced. All renal allograft recipients, with vascular complications, should undergo to PTA procedure as a first option.
\end{abstract}

\section{INTRODUCTION}

Despite high graft and recipient survival figures worldwide today, technical complications can threaten the transplant in the postoperative period. Vascular complications are commonly related to technical problems in establishing vascular continuity or to damage that occurs during donor nephrectomy or preservation [1]. Postoperative vascular complications in renal transplant recipients are important causes of graft dysfunction associated with high morbidity and mortality. Interventional approach plays a major role in the management of vascular complications in renal transplants, including arterial stenosis, arteriovenous fistulas (AVF), pseudoaneurysms (PA) and thrombosis [2]. Vascular complications are uncommon (1-3\%) in kidney transplantation, but recipient morbidities may result in loss of the allograft. Many studies report the significant influence of vascular complications on the graft's durability [3,4]. Early and middle-term vascular complications include external iliac artery iatrogenic or spontaneous lesions and drainage vein thrombosis. Major vascular complications including vascular anastomosis thrombosis, internal iliac artery stenosis, internal iliac artery rupture, obliterant vasculitis and external iliac vein rupture. Percutaneous transluminal angioplasty (PTA)

*Address correspondence to this author at the Institute of Cardiac Surgery, University of Bari, Bari, Italy; E-mail: abortone@cardiochir.uniba.it has been proposed as a valid, less invasive and alternative treatment to surgical repair in these patients.

Impaired arterial perfusion of the transplant may be the leading cause for graft dysfunction or refractory hypertension. Therefore, non-invasive imaging modalities are required to detect and locate vascular complications with high accuracy. Although Doppler ultrasonographic evaluation and gadolinium enhanced magnetic resonance angiography (MRA) are useful diagnostic screening methods, conventional angiography remains the gold standard procedure for final diagnosis, and also allows for endovascular treatment.

Minimally invasive techniques by PTA and stent placement have emerged as safe and effective procedures with a high technical success rate reducing substantial morbidity. They can be considered as an alternative to surgical treatment of transplant renal artery stenosis (TRAS).

\section{MATERIALS AND METHODS}

Between 1992 and 2007 we performed 724 renal transplantations (612 from cadaveric donors, 89 from living donors and 23 double kidney transplantations) in our Department. Five of these (4 from cadaveric donors and 1 from living donors) presented vascular complications in the post transplantation period. Two of them (from cadaveric donors) were treated by open surgery: 1 graft thrombosis character- 
ized by sudden anuria and by irreversible loss of renal function and treated with allograft nephrectomy and 1 arterial stenosis by acute and iatrogenic bending of the renal artery. In all patients an end-to-side anastomosis was performed between the transplanted renal artery and the external iliac artery of the recipient. The other 3 patients (from cadaveric donors) were treated by endovascular approach. One affected by dissection secondary to the site clamp and suture stenosis of the external iliac artery, 1 spontaneous pseudoaneurysm with uncontained rupture of the same artery and another with pseudoaneurysm at the site of previous transplantation. All patients were treated by endovascular PTA approach. After i.v. bolus administration of heparin (100 IU/Kg), a hydrophilic (0.035 inch) guide wire was advanced across the lesions and the appropriate device was deployed under a fluoroscopic control. Patients with pseudo-aneurysm were treated by self-expandable stent-graft PTFE endoprostheses
(Viabahn - Gore) release whereas in the other patient a balloon-expandable covered stent (Advanta V12 - Atrium) was used.

The first patient was a 62-years-old man affected by IgA glomerulonephritis with end-stage renal failure; in 2005 he underwent renal transplantation from a cadaveric donor. The patient achieved immediate graft function and the allograft was normally perfused. Four days later the patient underwent extravesical ureteral reanastomosis for distal ureteral necrosis. After 7 days the patient was discharged (serum creatinine $=1.0 \mathrm{mg} / \mathrm{dl}$ ). After 6 months, during follow-up visit, the patient mentions worsening of intermittent claudication (the patient was affected before transplantation). The MRangiography of the lower limbs, demonstrated stenosis followed by pseudoaneurysm arising from the external iliac artery at the site of iliac clamp detected by Angio CT scan and confirmed by angiography (Fig. 1a).
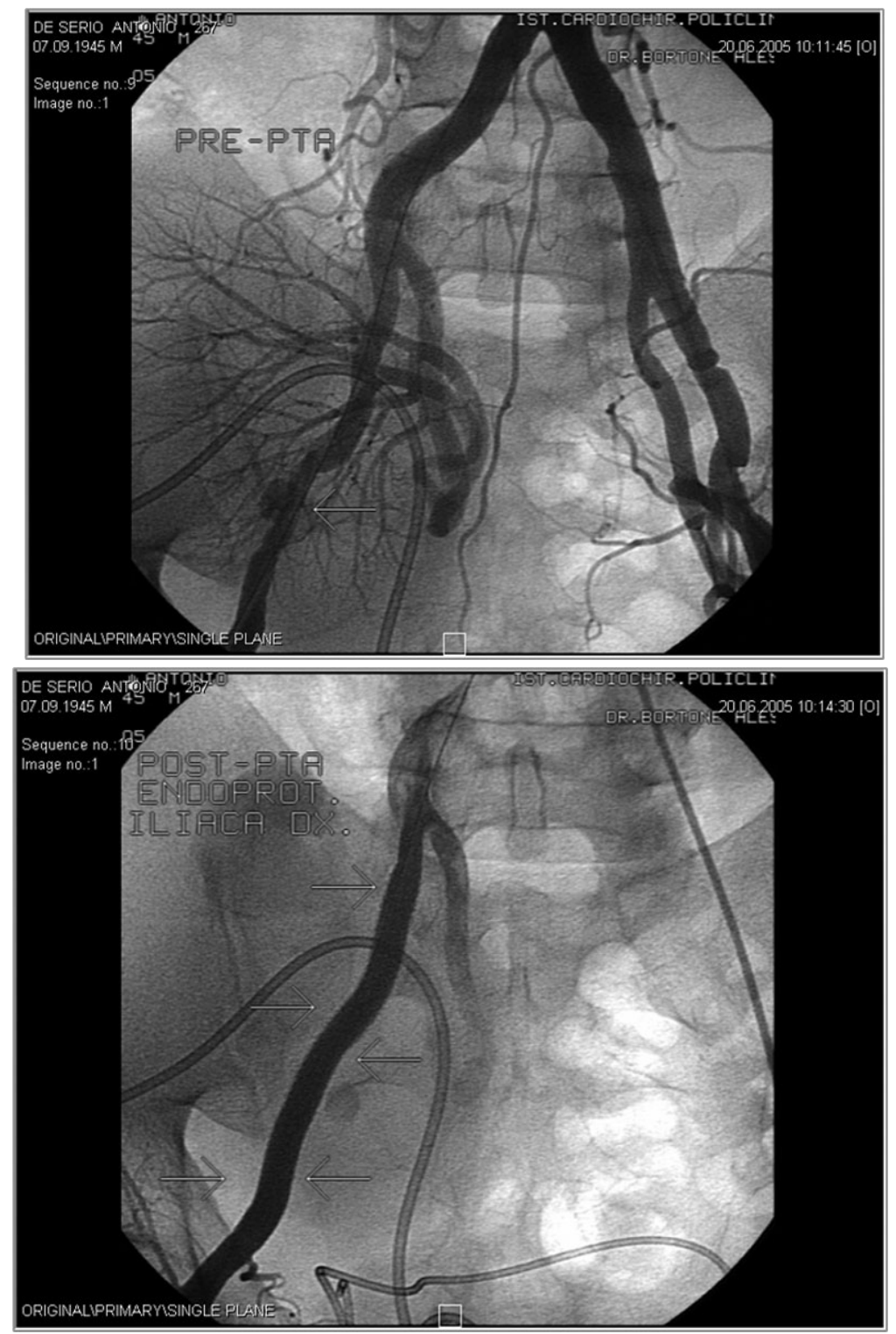

Fig. (1). (a) Angiographic study of a 62-year-old man with recent kidney transplantation admitted with an asymptomatic right common iliac artery pesudoaneurysm at the site of iliac clamp detected by Angio CT scan and confirmed by angiography. (b) Complete exclusion of pseudoanerysm by percutaneous implantation of Viabahn (GORE) endoprosthesis (8x100 mm). 
In the following days, the patient underwent PTA and placement of Viabahn (Gore) endoprosthesis $(8 \times 100 \mathrm{~mm})$ from right external iliac artery to the homolateral common femoral artery with optimal sealing and complete exclusion of the pseudoaneurysm; control angiography at the end of the procedure showed patency of homolateral internal iliac artery as well as of the 2 renal arteries of transplanted kidney (Fig. 1b)

The second patient, a 36-years-old man with congenital monorenal condition, was affected by vesico-ureteral reflux and Noonan's syndrome (Noonan syndrome (NS) is a phenotypically heterogeneous syndrome which is frequently associated with short stature, characterized by facial dysmorphism, congenital heart defects.) At the third month the patient underwent uretheroplasty and afterwards bladder neck plasty. In 1986 the patient started haemodialysis and 2 years later underwent the first kidney transplantation from a living donor (mother). In 1990 kidney explantation was necessary due to chronic allograft nephropathy. Four years later the patient underwent a second kidney transplantation from a cadaveric donor. In 1998 the patient started haemodialysis again and in the same year explantation of the kidney was performed. In March 2005, a iatrogenic left common iliac artery pesudoaneurysm at the site of iliac clamp was detected by Angio CT scan and confirmed by angiography (Fig. 2a). We decided to treat the lesion in the Cath Lab by percutaneous approach. Control angiography at the end of the procedure showed a complete exclusion of pseudoanerysm after percutaneous implantation of 2 Viabahn (Gore) endoprostheses $(7 \times 100 \mathrm{~mm}+8 \times 150 \mathrm{~mm})$ (Fig. 2b).

The third patient, a 61-year-old man, presented with acute renal failure and right leg ischemia 5 days after renal transplantation. The Doppler ultrasonographic evaluation demonstrated a reduction of renal flow and the angio-MRI confirmed a right external iliac artery dissection and stenosis at the proximal site of iliac clamp with patency of the renal and femoral Arteries. These findings were confirmed during angiographic study (Fig. 3a). A PTA with direct stenting of the right external iliac artery was then performed by double percutaneous femoral approach. A $6 \mathrm{~F}$ right Judkins catheter was advanced from the left femoral artery to the right common iliac artery in order to perform a control angiography and by antegrade approach a hydrophilic guide wire was advanced. Then embracement of the second hydrophilic guide wire through the retrograde advancement in right femoral artery was obtained (the embracement is a dedicated technique which allows to advance the second wire in a safely way because of the wire itself is inside the true lumen identified by the advancement of the first guide wire.) Finally, direct stenting of right external iliac artery was performed by covered stent Advanta V12 8 X $38 \mathrm{~mm}$ with patency of ipsilateral internal iliac, common femoral and renal arteries (Fig. 3b).

\section{RESULTS}

After percutaneous treatment clinical, biochemical, ultrasonographic and Doppler parameters showed a complete functional recovery of the ileo-femoral axis as well as of renal allograft function. All patients were discharged 5 days after the endovascular procedure. The follow-up was $100 \%$ complete in all patients. At an average follow-up of 12 a)

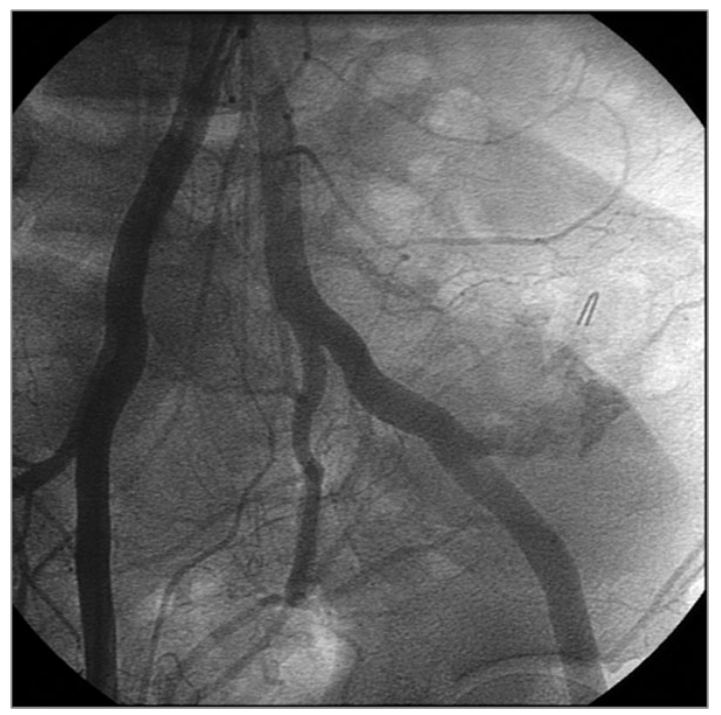

b)

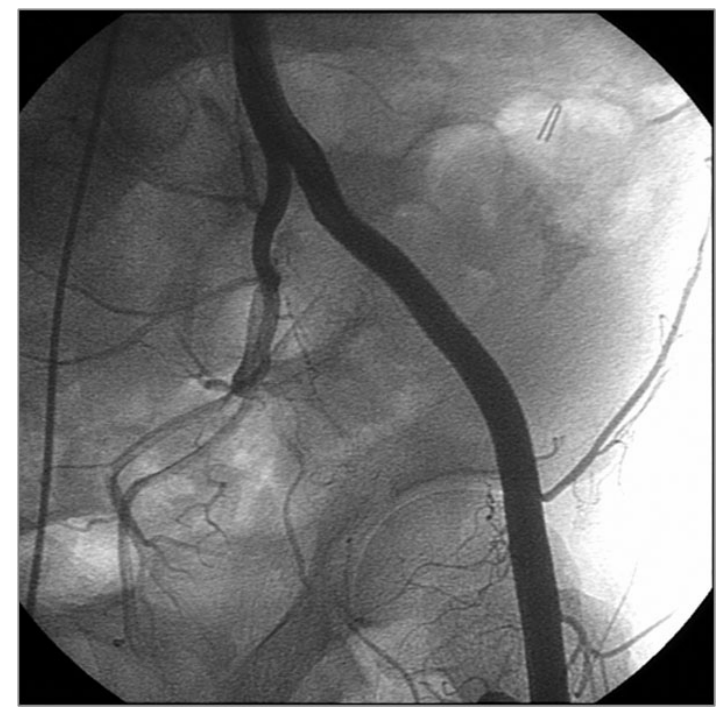

c)

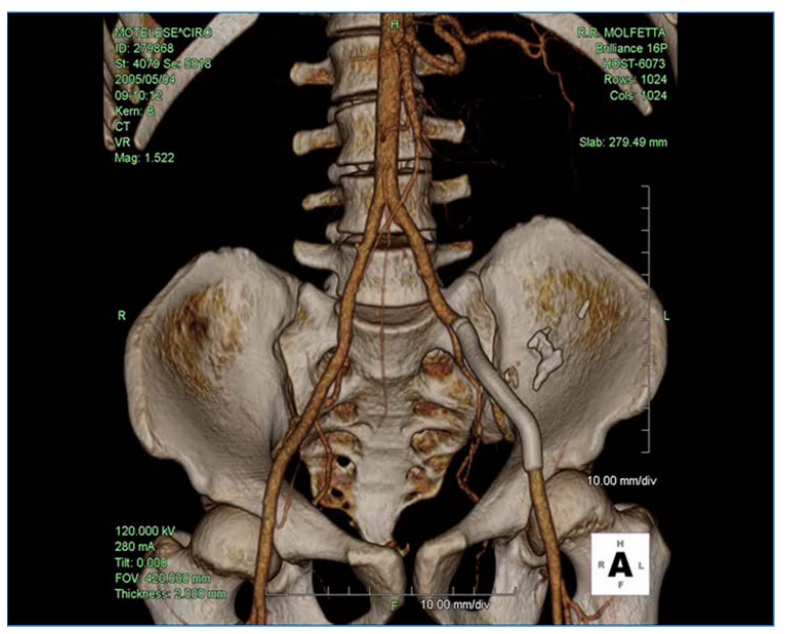

Fig. (2). (a) Angiographic study of a 36-year-old man with multiple kidney transplantations affected by left leg ischemia due to a iatrogenic left common iliac artery pesudoaneurysm at the site of iliac clamp detected by Angio CT scan and confirmed by angiography. (b) Control angiography: complete exclusion of pseudoanerysm by percutaneous implantation of Viabahn (GORE) endoprostheses $(7 \times 100 \mathrm{~mm}+8 \times 150 \mathrm{~mm})$. (c) 3D rendering by Angio-CT scan 1 month after the procedure. 
a)

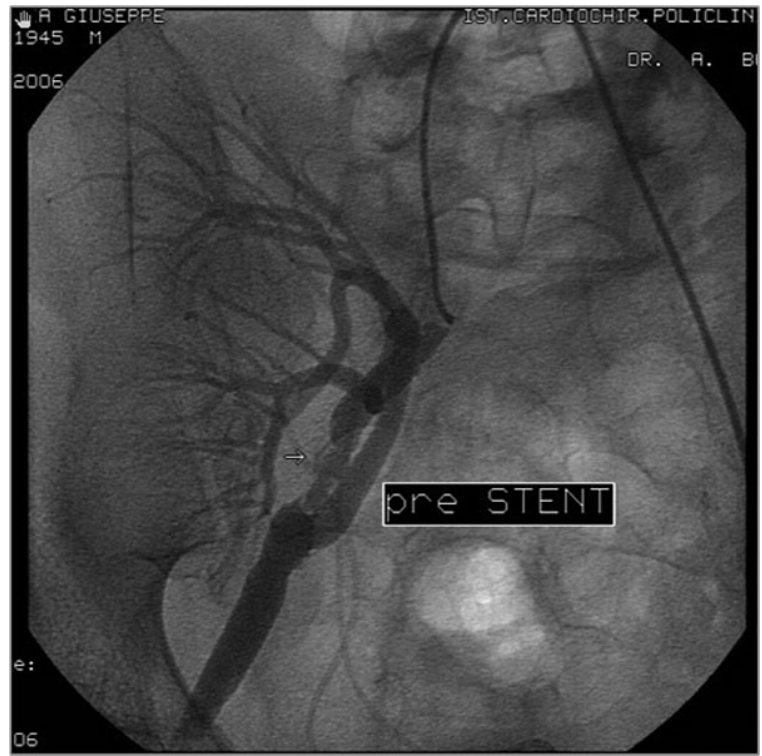

b)

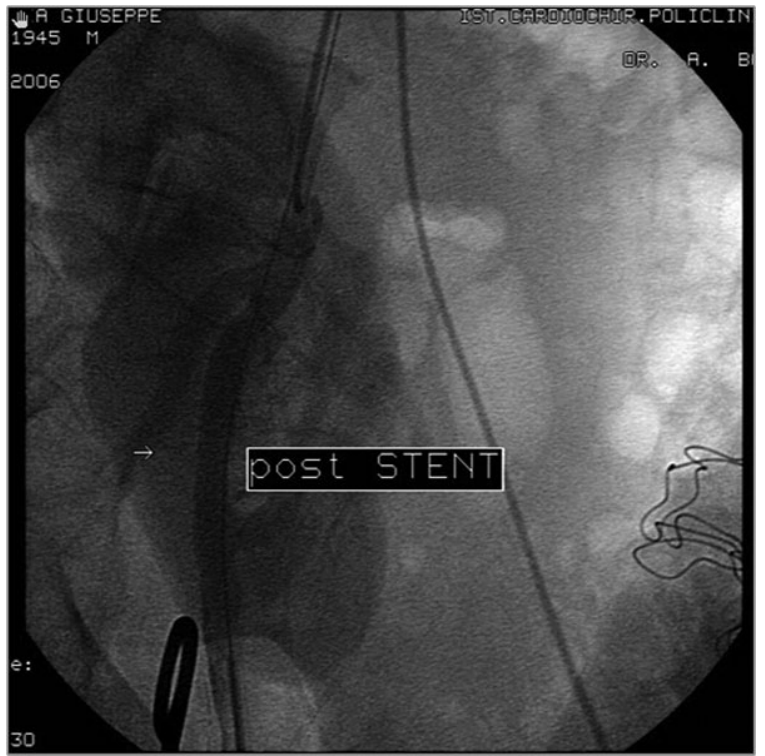

Fig. (3). (a). Angiographic study of a 61-year-old man with recent kidney transplantation affected by acute renal failure and right leg ischemia due to a reduction of flow by Eco- Color Doppler analysis. Right external iliac artery dissection and stenosis at the site of iliac clamp with patency of renal and femoral arteries by angiography. (b) PTA by percutaneous direct stenting of right external iliac artery.

months, by angio-CT scan, no recurrence of stenoses or pseudo-aneurysms were found.

\section{CONCLUSIONS}

Vascular and lymphatic complications are seen in less than $10 \%$ of renal transplant recipients, but they are an important cause of graft dysfunction. In contrast to other causes of transplant dysfunction, vascular complications have a high associated morbidity and mortality $[5,6]$. Once identified, vascular lesions are usually easily repaired [7]. Although angiography remains the gold standard for diagnosis and therapy of vascular complications, ultrasound and ColourDoppler modes are an excellent non invasive modality for evaluating the affected vessels.

PTA followed by stent placement is safe, effective and less invasive than surgical repair. Short and middle-term follow-up showed the benefit of the procedure, moreover the hospital stay is dramatically reduced. In our experience all renal allograft recipients, with vascular complications, should undergo PTA procedure as a first option in order to preserve renal allograft function and to avoid further complications. The early treatment of vascular complications is essential to preserve renal function and therefore, endovascular therapy represents our first choice.

\section{REFERENCES}

[1] Patel NH, Jindal RM, Wilkin T, et al. Renal arterial stenosis in renal allografts: retrospective study of predisposing factors and outcome after percutaneous transluminal angioplasty. Radiology 2001; 219: 665-7.

[2] Beecroft JR, Rajan DK, Clark WI, et al. Transplant renal artery stenosis: outcome after percutaneous intervention. J Vasc Interv Radiol 2004; 15: 1407-13.

[3] Asztalos L, Olvasztó S, Fedor R, et al. Renal artery aneurysm at the anastomosis after kidney transplantation. Transplant Proc 2006; 38 : 2915-18.

[4] Polak WG, Jezior D, Garcarek J, et al. Incidence and outcome of transplant renal artery stenosis: single center experience. Transplant Proc 2006; 38: 131-2.

[5] Emiroglu R, Karakayali H, Sevmis S, et al. Vascular complications in renal transplantation. Transplant Proc 2001; 33: 2685.

[6] Orlic P, Vukas D, Drescik I, et al. Vascular complications after 725 kidney transplantations during 3 decades. Transplant Proc 2003; 35: 1381 .

[7] Silvia RG, Lima VC, Amorim JE, et al. Angiplasty with stent is preferred therapy for postrasplant renal artery stenosis. Tansplant Proc 2002; 34: 514-5.

This is an open access article licensed under the terms of the Creative Commons Attribution Non-Commercial License (http://creativecommons.org/licenses/by-nc/3.0/) which permits unrestricted, non-commercial use, distribution and reproduction in any medium, provided the work is properly cited. 